\title{
Article
}

\section{Measuring maximal horizontal deceleration ability using radar technology: Reliability and sensitivity of kinematic and kinetic variables}

Harper, Damian James, Morin, Jean-Benoit, Carling, Christopher and Kiely, John

Available at http://clok.uclan.ac.uk/33971/

Harper, Damian James, Morin, Jean-Benoit, Carling, Christopher and Kiely, John ORCID: 0000-0001-9817-0224 (2020) Measuring maximal horizontal deceleration ability using radar technology: Reliability and sensitivity of kinematic and kinetic variables. Sports Biomechanics. ISSN 1476-3141

It is advisable to refer to the publisher's version if you intend to cite from the work. http://dx.doi.org/10.1080/14763141.2020.1792968

For more information about UCLan's research in this area go to http://www.uclan.ac.uk/researchgroups/ and search for <name of research Group>.

For information about Research generally at UCLan please go to http://www.uclan.ac.uk/research/

All outputs in CLoK are protected by Intellectual Property Rights law, including Copyright law. Copyright, IPR and Moral Rights for the works on this site are retained by the individual authors and/or other copyright owners. Terms and conditions for use of this material are defined in the policies page. 


\section{Measuring maximal horizontal deceleration ability using radar}

2 technology: Reliability and sensitivity of kinematic and kinetic

3 variables

4

5 Damian J. Harper ${ }^{1}$, Jean-Benoit Morin ${ }^{2}$, Christopher Carling ${ }^{3}$ and John

6 Kiely $^{1}$.

7 Institute of Coaching and Performance, School of Sport and Health Sciences,

8 University of Central Lancashire, Preston, UK; ${ }^{2}$ Côte d'Azur University, LAMHESS,

9 Nice, France; ${ }^{3}$ Centre for Elite Performance, French Football Federation, Paris, France.

12 Corresponding Author:

13 Name: Damian Harper

14 Address: School of Sport and Health Sciences, University of Central Lancashire,

15 Preston, PR1 2HE

16 Email: DJHarper@uclan.ac.uk

17 Twitter: @DHMov

19 Compliance with Ethical Standards

20 Funding

21 No sources of funding or financial support was received for the preparation of this

22 manuscript

23

24 Conflicts of interest

25 Damian J. Harper, Jean-Benoit Morin, Christopher Carling and John Kiely declare there

26 are no conflicts of interests that are directly relevant to the context of this article. 


\section{Measuring maximal horizontal deceleration ability using radar \\ 29 technology: Reliability and sensitivity of kinematic and kinetic variables}

Radar technology has potential for providing new insights into maximal horizontal deceleration ability. This study aimed to investigate the intra- and inter-day reliability and sensitivity of kinematic and kinetic variables obtained from a novel, maximal horizontal deceleration test, using radar technology. Thirty-eight university sport athletes completed testing for intra-day analysis. Twelve of these participants also completed the deceleration test on a second day for inter-day analysis. The maximal horizontal deceleration test required participants to decelerate maximally following $20 \mathrm{~m}$ maximal horizontal sprint acceleration. Reliability was assessed using the intraclass correlation coefficient (ICC) and coefficient of variation (CV\%). Sensitivity was evaluated by comparing typical error (TE) to smallest worthwhile change (SWC). A number of kinematic and kinetic variables had good (ICC $>0.75, \mathrm{CV}<10 \%$ ) overall intra-day reliability, and were sensitive to detect small-to-moderate changes in deceleration performance after a single familiarisation session. Only kinetic variables had good overall inter-day reliability and were sensitive to detect moderate changes in deceleration performance. Utilisation of this test protocol to assess maximal horizontal deceleration can provide new insights into individual maximal horizontal deceleration capabilities. Future work using this or similar approaches may 
Within competitive team sports contexts players must frequently and rapidly change velocity to dynamically adapt to evolving technical and tactical game demands. Such velocity changes can be positive (acceleration) or negative (deceleration), with both considered to be critical components of match-play performance. As illustrated in team sports such as soccer, players typically perform between 16-39 high-intensity accelerations $\left(>3 \mathrm{~m} / \mathrm{s}^{2}\right)$ and $43-59$ high-intensity decelerations $\left(<-3 \mathrm{~m} / \mathrm{s}^{2}\right)$ per match $(\mathrm{de}$ Hoyo et al., 2016; Russell et al., 2016; Tierney, Young, Clarke, \& Duncan, 2016). Furthermore, during the most demanding passages of play, players typically perform between 6.4 to 7.9 high-intensity accelerations and decelerations per minute (MartínGarcía, Casamichana, Gómez Díaz, Cos, \& Gabbett, 2018). Consequently, the capacity to profile individual players' maximal horizontal sprint acceleration and deceleration abilities, and subsequently apply these insights to inform the design of performance enhancement and injury prevention strategies, may be highly beneficial within team sports environments.

Sprint accelerations have been extensively researched, providing new insights into the technical and mechanical capabilities needed to accelerate rapidly (Colyer, Nagahara, Takai, \& Salo, 2018; Cross, Brughelli, Samozino, \& Morin, 2017). Subsequently, training interventions targeting specific components of acceleration, such as the capacity to generate a greater horizontal component of ground reaction force, have been designed and practically implemented (Morin, Edouard, \& Samozino, 2011; Morin et al., 2015; Morin \& Samozino, 2016). Crucially, however, far fewer investigations have documented player's ability to decelerate rapidly. As such, there is substantially less available evidence capable of informing training strategies targeting the development of rapid deceleration capabilities (Harper \& Kiely, 2018). This is problematic for sports science and medical professionals working with team sport athletes, where high intensity decelerations are typically performed more frequently than high intensity accelerations, and also inflict more negative consequences than equivalently intense accelerations (Harper, Carling, \& Kiely, 2019).

Indeed, in comparison to accelerations, rapid decelerations impose higher mechanical loads during match play (Dalen, Ingebrigtsen, Ettema, Hjelde, \& Wisløff,

87 2016) and result in a ground reaction force profile with significantly higher impact 
peaks and loading rates (Verheul et al., 2019). As such, there is an exacerbated risk of tissue damage and the likelihood of injury occurrence (Howatson \& Milak, 2009; Keane, Salicki, Goodall, Thomas, \& Howatson, 2015). Hence, the development of superior acceleration capabilities, if not accompanied by concurrently improving deceleration capabilities, could potentially lead to performance deficiencies in tasks that demand rapid decelerations from high approach velocities (Loturco et al., 2019). Accordingly, protocols capable of comprehensively and accurately profiling a player's ability to rapidly decelerate may provide important diagnostic information to help inform and guide performance enhancement and injury prevention training strategies.

Radar and laser devices are recommended for profiling horizontal sprint acceleration capabilities (Nagahara et al., 2017). Such devices could also be beneficially employed to profile maximal horizontal decelerations in more detail than previously possible (Simperingham, Cronin, \& Ross, 2016). For example, commonly estimated mechanical outputs, such as horizontal force and power, can be derived for sprint accelerations by applying simple computational methods based on Newtonian principles applied to the centre of mass (Morin, Samozino, Murata, Cross, \& Nagahara, 2019). Such metrics, potentially, provide valuable insights into the mechanical capabilities needed to decelerate rapidly. Only a small number of studies, however, have attempted to assess horizontal deceleration (Ashton \& Jones, 2019; Cesar \& Sigward, 2015, 2016; Graham-Smith, Rumpf, \& Jones, 2018; Harper, Jordan, \& Kiely, 2018; Naylor \& Greig, 2015). Furthermore, only one of these studies examined the reliability and sensitivity of a laser device to measure maximal horizontal deceleration abilities (Ashton \& Jones, 2019). However, this study only reported deceleration distances measured at 75, 50, 25 and $0 \%$ of the players maximal $15 \mathrm{~m}$ sprint velocity. Importantly, the trial-to-trial variability (measurement error) for all four of these variables was large ( $\mathrm{CV}>10 \%)$, making it difficult to detect small but meaningful changes in horizontal deceleration ability. The authors suggested that these large CV values could be due to inter-trial differences in when, and where, athletes commenced their decelerations. Consequently, it is feasible that regulating the velocity at which decelerations commence, as per previous work investigating maximal horizontal deceleration abilities (Harper et al., 2018), could improve the reliability and sensitivity of collated deceleration data.

Therefore, the aim of this study was to determine the intra- and inter-day reliability and sensitivity of radar-derived kinematic and kinetic measurements, 
121 obtained during maximal horizontal decelerations from a regulated running velocity. It

122 was hypothesised that a range of kinematic and kinetic variables would have good (ICC

$123>0.75, \mathrm{CV}<10 \%$ ) overall intra- and inter-day reliability, and would be sufficiently

124 sensitive to detect small-to-moderate changes in deceleration performance.

\section{Methods}

\section{Participants}

127 Thirty-eight university sport athletes ( $n=29$ male, $n=9$ female, age: $19.7 \pm 1.7$ years,

128 height: $176 \pm 10 \mathrm{~cm}$, body mass: $73.0 \pm 14.7 \mathrm{~kg}$ ) engaging primarily in team sports

129 (soccer, rugby league, rugby union, netball) volunteered to participate. The eligibility

130 criteria specified, that for inclusion in the study, all participants had to be regularly

131 partaking (3 times per week) in moderate to high intensity exercise, and be familiar with

132 change of direction (COD) tasks requiring high intensity accelerations and

133 decelerations. Participants who had suffered musculoskeletal injury, that had prevented

134 participation in sport or physical activity within the previous 3 months, were excluded.

135 Testing was conducted mid-way through the UK University competitive sport season.

136 All participants completed testing on day 1 (intra-day analysis), whilst twelve

137 participants ( $n=8$ male, $n=4$ female, age: $19.4 \pm 1.5$ years, height: $175 \pm 10 \mathrm{~cm}$, body

138 mass: $74.4 \pm 14.3 \mathrm{~kg}$ ) also completed testing on day 2 (inter-day analysis). The

139 institutional ethics review committee at the University of Central Lancashire granted

140 ethical approval. All participants were provided with a written information sheet that

141 explained the requirements of the study, and the benefits and risks of participation.

142 Participants were also given opportunity to ask any questions before providing

143 voluntary informed written consent.

\section{Experimental design}

145 A within-subject repeated measures research design was used to determine the

146 intra- and inter-test reliability of kinetic and kinematic variables obtained from a

147 new test of maximal horizontal deceleration measuring using radar technology.

148 All experimental procedures took place over a 2-week period, in which

149 participants were required to complete 3 testing sessions with at least 48 hours

150 recovery between. In the first test session all participants had anthropometric

151 measurements taken and completed a $20 \mathrm{~m}$ horizontal sprint. They were then 
152 familiarised with the protocols of the maximal horizontal deceleration test.

153 Familiarisation involved participants firstly observing a demonstration of the

154 maximal horizontal deceleration test. Following this all participants practiced the

155 deceleration test following a progressive increase in intensity $(70,80,100 \%$

156 perceived effort). In the subsequent 2 sessions participants completed the maximal

157 horizontal deceleration test to allow determination of intra- and inter-test

158 reliability. Prior to all testing participants completed the same 15-minute

159 standardised warm-up that included forward and backward jogging, dynamic

160 stretching, and 3 practice trials of the deceleration test following a progressive

161 increase in intensity (70,80 and $100 \%$ perceived effort). To reduce the potential

162 influence of confounding variables all sessions were completed at the same time

163 of the day (9:00am to 12:00pm) on an indoor artificial sports surface.

164 Furthermore, the same accredited sport and exercise scientist administered all test

165 instructions, and measurements, and conducted subsequent data analysis.

\section{Procedures}

168 Anthropometrics

169 Standing height was measured to the nearest $\mathrm{cm}$ using a stadiometer (Seca 217,

170 Hamburg, Germany), and body mass to the nearest $0.1 \mathrm{~kg}$ using electronic weighing scales (Seca, Hamburg, Germany).

Maximal Horizontal Sprint Test

174 Sprints times were recorded over $20 \mathrm{~m}$ distance using timing gates (Witty, Microgate,

175 Bolzano, Italy) set to a height of $0.8 \mathrm{~m}$ (Cronin \& Templeton, 2008). Times were

176 recorded to the nearest $0.01 \mathrm{~s}$. Each sprint commenced from a stationary split stance

177 position with the front foot positioned $30 \mathrm{~cm}$ behind the timing gate to prevent a false

178 trigger. Participants were instructed to initiate their own start with no backward step or

179 'rocking motion' and to sprint as fast as possible. Each participant was allowed 2 trials

180 interspersed by a passive recovery period of at least a 2-minutes duration. The best $20 \mathrm{~m}$

181 split was used as a 'criterion' time in the maximal horizontal deceleration test.

184 Maximal horizontal deceleration was assessed using an acceleration-deceleration ability

185 (ADA) test (Harper et al., 2018). Participants were instructed to use the same start 
186 protocol employed for the horizontal sprint test and to sprint maximally over $20 \mathrm{~m}$

187 before performing a maximal horizontal deceleration. The $20 \mathrm{~m}$ point marking the start 188 of the deceleration phase was identified with tall marker poles. Immediately following 189 the end of the deceleration, players backpedalled to the $20 \mathrm{~m}$ line. This created a clear

190 change in velocity on the instantaneous velocity-time graph captured by the radar

191 device, and enabled the end of the deceleration phase to be easily identified (Figure 1).

192 To ensure the start of the deceleration commenced as close to the $20 \mathrm{~m}$ point as

193 possible, any $20 \mathrm{~m}$ time that was $5 \%$ greater than the best $20 \mathrm{~m}$ split time achieved

194 during the horizontal sprint test was considered as an unsuccessful trial. In such cases

195 the participant was asked to repeat the test following at least a 3-minute recovery

196 period. Participants were asked to perform a maximum of 5-trials, with the 2 successful

197 trials with the highest average deceleration used for analysis.

<INSERT FIGURE 1 ABOUT HERE> using a radar device (Stalker ATS II, Applied Concepts, Inc., Dallas, TX, USA) sampling at $47 \mathrm{~Hz}$, which was connected to a laptop with the Stalker ATS system software (Version 5.0, Applied Concepts, Inc., Dallas, TX, USA) for data acquisition. To enable instantaneous horizontal velocity to be recorded whilst participant was moving away (acceleration and deceleration phases) and towards (backpedal to signify end of deceleration phase) the radar, the target direction setting on the radar was set to 'both'. The radar device was mounted on a heavy-duty tripod and positioned $5 \mathrm{~m}$ behind the start line, which is within the 4.6 to $9.6 \mathrm{~m}$ distance recommended by the manufacturer for recording acceleration and braking run tests. The radar device was set to a height $1 \mathrm{~m}$ above the ground to approximately align with the participant's centre of

212 mass. When the participant was in the stationary start position, data recording was

213 started using the 'any key' feature of the Stalker ATS system software, and a verbal

214 instruction of 'when you are ready' provided to the participant. Data capture was ended

215 using the 'any key' feature once the participant had backpedalled to the $20 \mathrm{~m}$ line

216 following the maximal horizontal deceleration. 
219 All data was manually processed in the graph mode editor of the Stalker ATS software

220 following similar procedures outlined by Simperingham et al. (2017) for horizontal

221 force-velocity-power profiling during short sprint-running acceleration. This involved:

222 (i) deleting all data recorded before the start of the sprint and following the end of the

223 deceleration phase, (ii) nominating all trials to be 'acceleration runs' thereby forcing the

224 start of the velocity-time curve through the zero point, (iii) applying a digital fourth

225 order, zero lag Butterworth filter (as recommended by the manufacturer), and (iii)

226 manually removing unexpected high and low data points on the velocity-time curve that

227 were likely caused by segmental movements of the participants while sprinting. Once

228 manual processing had been completed instantaneous horizontal velocity $(v)$, time $(t)$

229 and distance $(d)$ for each trial was exported to Microsoft Excel for further analyses.

230 The start of the deceleration phase was defined as the time point immediately

231 following the maximum velocity $\left(\mathrm{V}_{\max }\right)$ achieved during the $20 \mathrm{~m}$ sprint. The end of the

232 deceleration phase was defined as the lowest velocity ( $\mathrm{V}_{\text {low }}$ ) following $\mathrm{V}_{\max }$. The

233 deceleration phase was also further divided into early and late deceleration phases by

234 using the time point associated with $50 \% \mathrm{~V}_{\max }$ (Figure 2).

235

236

< INSERT FIGURE 2 ABOUT HERE >

237

Instantaneous horizontal deceleration was calculated between each data point captured across the entire deceleration phase using the following equation:

$$
\text { Deceleration }\left(m / s^{2}\right)=\frac{\left(v_{f}-v_{i}\right)}{\left(t_{f}-t_{i}\right)}
$$

240 Where $v$ is the velocity, $t$ is the time, $f$ is the final velocity or time, and $i$ is the initial 241 velocity or time.

242 Kinematic variables analyzed included: (1) average deceleration $\left(\mathrm{DEC}_{\mathrm{ave}}\right.$;

243 average of all instantaneous deceleration values calculated from start to end of

244 deceleration phase), (2) maximum deceleration ( $\mathrm{DEC}_{\max }$; highest instantaneous

245 deceleration value calculated between start and end of deceleration phase), (3) early-

246 deceleration (E-DEC; average of all instantaneous deceleration values calculated

247 between start of deceleration phase to $50 \% \mathrm{~V}_{\max }$, (4) late-deceleration (L-DEC; average

248 of all instantaneous deceleration values calculated between $50 \% \mathrm{~V}_{\max }$ and end of

249 deceleration phase), (5) time to stop (TTS; time taken from start to end of deceleration

250 phase), (6) time to $50 \% \mathrm{~V}_{\max }\left(\mathrm{TT} 50 \% \mathrm{~V}_{\max }\right.$; time taken from the start of the deceleration 
251 phase to $50 \% \mathrm{~V}_{\max }$ ) and (7) distance to stop (DTS; distance travelled from start to end 252 of deceleration phase).

253 Kinetic variables estimated in the deceleration phase included average horizontal

254 braking force $\left(\mathrm{HBF}_{\mathrm{ave}}\right)$, braking power $\left(\mathrm{HBP}_{\mathrm{ave}}\right)$ and braking impulse $\left(\mathrm{HBI}_{\mathrm{ave}}\right)$

255 calculated using the average of all instantaneous HBF, HBI and HBP values obtained

256 from start to end of deceleration. Also estimated were the average HBF, HBP and HBI

257 during the early and late deceleration phases using instantaneous values obtained

258 between the start of deceleration and $50 \% \mathrm{~V}_{\max }$, and $50 \% \mathrm{~V}_{\max }$ and end of deceleration,

259 respectively. Maximal HBF, HBP and HBI were calculated using the highest

260 instantaneous value between start and end of deceleration phase.

261

262

Instantaneous HBF was calculated between each data point during the

263 deceleration phase using Newton's second law of motion:

264

$$
\operatorname{HBF}(t)=[m \times a(t)]+\mathrm{F}_{\text {air }}(t)
$$

265 Where $m$ is the body mass of the participant and $\mathrm{F}_{\text {air }}$ is the air friction, which is influenced by the frontal area of the participant (Af) (Morin et al., 2019):

267

$$
\mathrm{A}_{\mathrm{f}}=\left(0.2025 \times \text { height }^{0.725} \times \text { mass }^{0.425}\right) \times 0.266
$$

Instantaneous HBP was calculated between each data point during the deceleration phase using the product of HBF and $v$ :

271

$$
\mathrm{HBP}=\mathrm{HBF} \times v
$$

272

273 Instantaneous HBI was calculated between each data point during the deceleration phase

274 using change in momentum:

275

$$
J(t)=M_{f}-M_{i}
$$

276 Where $\mathrm{J}$ is the impulse, $\mathrm{M}_{\mathrm{f}}$ is the final momentum and $\mathrm{M}_{\mathrm{i}}$ is the initial momentum. Instantaneous momentum was calculated using the following equation: 


$$
\text { Momentum }(\mathrm{t})=\mathrm{v} \times \text { mass }
$$

279

280

281

282

283

284

285

286

287

288

289

290

291

292

293

294

295

296

297

298

299

300

301

302

303

304

305

306

307

308

309

310

\section{Statistical analysis}

The mean \pm SD was calculated for all radar derived variables. Intra- and inter-day reliability was calculated by determining the relative (intra-class correlation coefficient; ICC) and absolute (coefficient of variation; CV\%) reliability using the 'consecutive pairwise' Microsoft Excel spreadsheet (Hopkins, 2015). This spreadsheet uses the ICC $(3,1)$, which provides the correlation expected between pairs of measurements in any two trials, when all participants have performed the same two trials. CV was calculated from the TE, and expressed as a \%. The thresholds used to interpret the ICC were taken from guidelines (Koo \& Li, 2016) for reporting ICC values: $\leq 0.49=$ poor; 0.50 to 0.74 $=$ moderate $; 0.75$ to $0.89=$ good $; \geq 0.90=$ excellent. The $\mathrm{CV} \%$ was interpreted using the following scale (McMahon, Lake, \& Comfort, 2018): > 15 poor; 10 to 15 moderate; 5 to 10 good; < 5 excellent. Overall reliability was interpreted by combining both the ICC and the CV\% scales as follows: ICC > 0.9 and CV\% $<5=$ excellent; ICC 0.75 to 0.9 and $\mathrm{CV} \%<10=$ good; $\mathrm{ICC}<0.75$ or $\mathrm{CV} \%>10=$ moderate; $\mathrm{ICC}<0.75$ and $\mathrm{CV} \%$ $<10=$ poor. The $90 \%$ confidence intervals for all reliability results were also included. To determine the sensitivity of each radar derived variable the raw TE obtained from the Microsoft Excel spreadsheet (Hopkins, 2015) was compared to the smallest worthwhile change (SWC). SWC was calculated by multiplying the between-subject SD by $0.2\left(\mathrm{SWC}_{0.2}\right)$, which is a small effect, or by $0.5\left(\mathrm{SWC}_{0.5}\right)$, which is an alternative moderate effect. If the TE was less than the SWC the test variable was rated as 'good', if the TE was equal to the SWC it was rated as 'OK', and if the TE was higher than the SWC it was rated 'poor'.

\section{Results}

\section{Intra-day reliability and sensitivity}

The mean and standard deviation for all kinematic and kinetic variables connected with the best 2 average deceleration trials on day 1 of testing are shown in table 1 . The corresponding ICC and CV\% values to determine intra-day reliability, and the TE and SWC to determine the sensitivity of each test variable are also shown in table 1 . Of the kinematic variables only $V_{\max }$ had excellent $(\mathrm{ICC}=0.97, \mathrm{CV}=1.4 \%$ ) overall intra-test reliability, and was able to detect the $\mathrm{SWC}_{0.2}$. TT50\% $V_{\max }(\mathrm{ICC}=0.76, \mathrm{CV}=8 \%)$, TTS $(\mathrm{ICC}=0.82, \mathrm{CV}=5.3 \%), \mathrm{DTS}(\mathrm{ICC}=0.76, \mathrm{CV}=7.2 \%), \mathrm{DEC}_{\mathrm{ave}}(\mathrm{ICC}=0.87, \mathrm{CV}=$ $5.2 \%)$ and $\mathrm{E}_{-\mathrm{DEC}} \mathrm{ave}(\mathrm{ICC}=0.76, \mathrm{CV}=8.8 \%)$ had good overall intra-test reliability. 
311 However, only TTS and $\mathrm{DEC}_{\text {ave }}$ demonstrated sufficient sensitivity to detect the

$312 \mathrm{SWC}_{0.5}$, with $\mathrm{TT} 50 \% V_{\max }$, DTS and $\mathrm{E}^{-\mathrm{DEC}_{\mathrm{ave}}}$ rated as ' $\mathrm{OK}$ '.

313 All kinetic variables apart from L-HBP ave, $\mathrm{HBF}_{\max }$ and $\mathrm{HBP}_{\max }$ had good (ICC >

$3140.8, \mathrm{CV}<10 \%$ ) overall reliability. However, only $\mathrm{HBP}_{\text {ave }}$ had sufficient sensitivity to

315 detect the $\mathrm{SWC}_{0.2}$. All kinetic variables were sensitive to detect $\mathrm{SWC}_{0.5}$.

316

\section{Inter-day reliability and sensitivity}

318 The mean and standard deviation for all kinematic and kinetic variables from day 1 and

319 day 2 of testing are shown in table 2. The corresponding ICC and CV\% values to

320 determine inter-test reliability, and the TE and SWC to determine the sensitivity of each variable across days are also shown in table 2 . Similar to intra-day reliability for the kinematic variables, only $V_{\max }$ had excellent $(\mathrm{ICC}=0.96, \mathrm{CV}=1.7 \%$ ) overall inter-day reliability, and was able to detect the $\mathrm{SWC}_{0.2}$. TTS $(\mathrm{ICC}=0.45, \mathrm{CV}=8.2 \%), \mathrm{DEC}_{\text {ave }}$ $(\mathrm{ICC}=0.73, \mathrm{CV}=8.0 \%)$ and $\mathrm{DEC}_{\max }(\mathrm{ICC}=0.61, \mathrm{CV}=7.9 \%)$ had moderate overall inter-day reliability. All other kinematic variables had poor $(\mathrm{ICC}=<0.75, \mathrm{CV}>10 \%)$ inter-day reliability.

For the kinetic variables $\mathrm{HBF}_{\mathrm{ave}}(\mathrm{ICC}=0.90, \mathrm{CV}=9.3 \%), \mathrm{HBP}_{\mathrm{ave}}(\mathrm{ICC}=0.93$, $\mathrm{CV}=8.9 \%)$ and $\mathrm{HBI}_{\mathrm{ave}}(\mathrm{ICC}=0.90, \mathrm{CV}=9.0 \%)$ had overall good inter-day reliability. However, only $\mathrm{HBP}_{\text {ave }}$ and $\mathrm{HBI}_{\mathrm{ave}}$ were sensitive to detect the $\mathrm{SWC}_{0.5} . \mathrm{HBF}_{\max }(\mathrm{ICC}=$ $0.89, \mathrm{CV}=8.2 \%), \mathrm{HBP}_{\max }(\mathrm{ICC}=0.96, \mathrm{CV}=6.2 \%)$ and $\mathrm{HBI}_{\max }(\mathrm{ICC}=0.90, \mathrm{CV}=$ $8.2 \%$ ) also had good overall inter-day reliability, and were sensitive enough to detect the $\mathrm{SWC}_{0.5}$. Both $\mathrm{E}-\mathrm{HBF}_{\text {ave }}(\mathrm{ICC}=0.89, \mathrm{CV}=12.2)$ and $\mathrm{L}-\mathrm{HBF}_{\mathrm{ave}}(\mathrm{ICC}=0.76, \mathrm{CV}=$ 11.7) had moderate inter-day reliability, and were sensitive enough to detect $\mathrm{SWC}_{0.5}$. Similarly, both E-HBI ${ }_{\mathrm{ave}}(\mathrm{ICC}=0.87, \mathrm{CV}=8.2 \%)$ and $\mathrm{L}-\mathrm{HBI}_{\mathrm{ave}}(\mathrm{ICC}=0.77, \mathrm{CV}=$ $11.4 \%$ ) had moderate inter-day reliability, although only E-HBI $\mathrm{Hve}_{\text {was }}$ sensitive to detect the $\mathrm{SWC}_{0.5}$.

\section{Discussion and Implications}

339 To our knowledge, this is the first study to examine the intra- and inter-day reliability and sensitivity of radar-derived kinematic and kinetic variables measured during a novel maximal horizontal deceleration test. The major findings of this study are: (1) a number of kinematic (i.e. TT50\% $V_{\max }$, TTS, DTS, $\mathrm{DEC}_{\mathrm{ave}}, \mathrm{E}-\mathrm{DEC}_{\mathrm{ave}}$ ) and kinetic (i.e. $\mathrm{HBF}_{\mathrm{ave}}$, $\mathrm{HBP}_{\mathrm{ave}}, \mathrm{HBI}_{\mathrm{ave}}, \mathrm{HBI}_{\mathrm{max}}$ ) variables had good overall intra-day reliability, and were 
sensitive to detect moderate changes in performance, (2) kinematic variables (TTS,

$345 \mathrm{DEC}_{\mathrm{ave}}$ and $\mathrm{DEC}_{\max }$ ) had moderate overall inter-day reliability, and (3) only kinetic 346 variables $\left(\mathrm{HBF}_{\mathrm{ave}}, \mathrm{HBP}_{\mathrm{ave}}, \mathrm{HBI}_{\mathrm{ave}}, \mathrm{HBF}_{\max }, \mathrm{HBP}_{\max }\right.$, and $\left.\mathrm{HBI}_{\max }\right)$ had good overall

347 inter-day reliability, and were adequately sensitive to detect moderate changes in

348 performance. Therefore, the original study hypothesis can be rejected, since only kinetic variables had good overall intra- and inter-day reliability, and were sufficiently sensitive to detect small-to-moderate changes in horizontal deceleration ability.

Previous studies measuring deceleration performance have used either a COD

352 (Hader, Mendez-Villanueva, Palazzi, Ahmaidi, \& Buchheit, 2016; Hader, Palazzi, \&

353 Buchheit, 2015; Jones, Thomas, Dos'Santos, McMahon, \& Graham-Smith, 2017) or

354 horizontal sprint acceleration-to-deceleration task (Ashton \& Jones, 2019; Cesar \&

355 Sigward, 2015, 2016; Graham-Smith et al., 2018; Harper et al., 2018; Naylor \& Greig,

356 2015). The use of a horizontal sprint acceleration to deceleration task allows

357 deceleration performance to be examined independently of COD-imposed technical

358 constraints. Furthermore, the deceleration phase during a COD task typically occurs

359 from sub-maximal sprinting velocities (Dos'Santos, Thomas, Comfort, \& Jones, 2018;

360 Hader et al., 2015), and subsequently may be unreflective of the deceleration

361 characteristics necessary to successfully decelerate from near-maximum sprint

362 velocities. Accordingly, during COD-related deceleration tasks, the deceleration

363 challenge may not be a valid representation of a performer's maximal deceleration 364 capacity.

Whilst a number of previous studies have used a horizontal sprint accelerationto-deceleration task to examine maximal deceleration capabilities, only one of these studies examined the reliability and sensitivity of the measures obtained (Ashton \& Jones, 2019). Here, deceleration performance was measured using a laser device following $15 \mathrm{~m}$ sprint acceleration, and evaluated using the deceleration distance measured at 75, 50, 25 and $0 \%$ of the participant's $15 \mathrm{~m}$ horizontal sprint velocity.

371 Based on their findings, the authors subsequently suggested using total DTS (0\% of 15

$372 \mathrm{~m}$ velocity) to measure deceleration ability. However, due to the higher average $\mathrm{CV}$

$373(10.52 \%)$ for this variable, it was also recommended that further work to be conducted

374 to establish a protocol that is more sensitive to tracking changes in horizontal

375 deceleration ability. It is likely, as suggested by the authors of this study, that the high

376 measurement variability, using this protocol, was due to the start of the deceleration

377 phase being defined as the velocity at the $15 \mathrm{~m}$ mark. For instance, the average $15 \mathrm{~m}$ 
378

379

380

381

382

383

384

385

386

387

388

389

390

391

392

393

394

395

396

397

398

399

400

401

402

403

404

405

406

407

408

409

410

411

velocity was $5.39 \mathrm{~m} / \mathrm{s}$, which was much lower than the average peak velocity $(6.84 \mathrm{~m} / \mathrm{s})$ recorded during the test. This finding implies that participants had already started to decelerate prior to the $15 \mathrm{~m}$ mark. To overcome this problem, in the current study, we defined the start of the deceleration phase as the time point immediately following $\mathrm{V}_{\max }$ achieved during the $20 \mathrm{~m}$ sprint. This definition has previously been used to quantify deceleration ability using a laser device (Graham-Smith et al., 2018). Furthermore, in order to reduce the likelihood of participants commencing deceleration prior to the $20 \mathrm{~m}$ mark, and to ensure better precision and consistency in when the deceleration phase commenced, any $20 \mathrm{~m}$ time that was $5 \%$ greater than the participants $20 \mathrm{~m}$ linear sprint time (without a maximal deceleration) was considered an unsuccessful trial. Using this criteria the average distance at which deceleration commenced was $17.2 \mathrm{~m}$, with excellent $(3.7 \%)$ to good $(5.9 \%)$ consistency demonstrated between trials and between days, respectively. Therefore, these findings demonstrate that by using $\mathrm{V}_{\max }$ to denote the start of deceleration, and by regulating the time at which deceleration commenced, consistent distances at which deceleration commences can be obtained.

The DTS variable in the present study had good overall intra-day reliability ( $\mathrm{ICC}=0.76, \mathrm{CV}=7.2 \%)$, but poor inter-day reliability $(\mathrm{ICC}=0.45, \mathrm{CV}=10.8 \%)$. The kinematic variable with the best intra- and inter-day reliability and sensitivity was $\mathrm{DEC}_{\mathrm{ave}}$. The overall reliability of this variable was good $(\mathrm{ICC}=0.87, \mathrm{CV}=5.2 \%)$ to moderate $(\mathrm{ICC}=0.73, \mathrm{CV}=8.0 \%$ ), with the sensitivity to detect small changes in performance rated as 'good', for intra-day reliability. These findings are similar to those of Varley, Fairweather, \& Aughey (2012), who reported a CV of $6 \%$ for $\mathrm{DEC}_{\text {ave }}$ when the deceleration phase was measured using a $10 \mathrm{~Hz}$ global positioning system during a horizontal running task performed from velocities ranging between 5 and $8 \mathrm{~m} / \mathrm{s}$. In the present study decelerations commenced from a much narrower velocity range (7.17 to $7.36 \mathrm{~m} / \mathrm{s})$ and were measured using a higher sampling rate $(47 \mathrm{~Hz})$. In the study by Varley et al. (2012) the rate of deceleration was not reported. Therefore, the similar CV\% found between these studies is likely due to the higher rates of deceleration (-4.36 to $-4.44 \mathrm{~m} / \mathrm{s}^{2}$ ) performed in the present study. Nonetheless, based on the findings of the present study, $\mathrm{DEC}_{\mathrm{ave}}$ is the kinematic variable of choice when monitoring SWC in maximal horizontal deceleration ability.

In sprint acceleration research, laser, radar and video devices are commonly used, in conjunction with using simple computational methods, to provide advanced insights into the mechanical (kinetic) determinants of sprint acceleration performance 
412 (Morin et al., 2019; Romero-Franco et al., 2017; Simperingham et al., 2016). Such an

413 approach provides a more in-depth understanding of the underpinning mechanical

414 features determining maximal sprint acceleration performance, and can be subsequently

415 used to inform individualised and specific training prescriptions (Morin \& Samozino,

416 2016). Despite widespread use in sprint acceleration profiling, this is the first study to

417 use instantaneous horizontal velocity-time data to estimate the horizontal braking force

418 (HBF), power (HBP) and impulse (HBI) during a maximal horizontal deceleration task.

419 The findings of this study show that, when averaged across the entire deceleration

420 phase, HBF, HBP and HBI had good overall intra-day (ICC $=0.95$ to $0.96, \mathrm{CV}=5.1$ to

$4215.7 \%$ ) and inter-day reliability (ICC $=0.90$ to $0.93, \mathrm{CV}=8.9$ to $9.3 \%$ ), and were

422 sufficiently sensitive to detect moderate changes in horizontal deceleration ability.

423 Subsequently, as is the case with horizontal sprint acceleration profiling,

424 coaches and sport science professionals can productively use these mechanical outputs

425 to obtain more in-depth understanding of their athlete's deceleration capabilities. In

426 different athletic context, such as rugby and American Football, within which players

427 operating in different positions typically have widely varying body masses, changes in

428 whole-body momentum — referred to in this study as the horizontal braking impulse

429 (HBI) - could provide particularly insightful information. Especially since these players

430 will inevitably have to generate higher braking forces in order to reduce higher whole-

431 body momentums. Future research should investigate the influence of these mechanical

432 variables on maximal deceleration performance capacities (e.g. average deceleration),

433 and compare the validity of these variables to direct measures obtained from embedded

434 force platforms.

435 In order to obtain a more thorough evaluation of deceleration performance, the

436 deceleration velocity profile was sub-divided into 'early' and 'late' deceleration phases.

437 It has previously been shown in walking gait termination that decelerating can involve

438 distinct phases: 'preparatory brake', 'fast brake' and 'final brake' (Jian, Winter, Ishac,

439 \& Gilchrist, 1993). The 'fast brake' period comprising a rapid reduction in velocity with

440 greater braking forces, whereas the 'final brake' comprised a small reduction in

441 velocity, with the main goal being to stabilise the centre of mass above the base of

442 support. By examining both the early and late deceleration phases, it is subsequently

443 possible to calculate a horizontal deceleration or braking-ratio, which could allow

444 further identification of individual-specific deceleration strategies and training needs. In

445 the present study, only HBF and HBI variables had good overall intra-day reliability 
446 ( $\mathrm{ICC}=0.84$ to $0.91, \mathrm{CV}=8.7$ to $9.6 \%$ ), and were sensitive enough to detect moderate 447 changes in the early and late deceleration phases. Furthermore, both of these variables 448 had moderate overall inter-day reliability ( $\mathrm{ICC}=0.76$ to $0.87, \mathrm{CV}=11.4$ to $12.2 \%$ ) and 449 were able to detect moderate changes in the early deceleration phase. Subsequently, for 450 the purpose of monitoring the early and late deceleration phases, the kinetic variables $451 \mathrm{HBF}$ and HBI are recommended. Further research is required to investigate the 452 importance of the early and late deceleration phases on overall deceleration 453 performance, and the neuromuscular performance characteristics that may contribute to superior early and late deceleration performance.

This study has limitations similar to those highlighted in previous work examining the reliability of horizontal force-velocity power profiling during short sprint accelerations (Simperingham et al., 2019). Specifically, raw data captured from the radar was filtered using the manufacturers own proprietary software. Therefore, it is possible that alternative post-processing methods may be more applicable. For example, analysing the raw data points using a rolling average across different time frames (e.g. $0.2,0.3 \mathrm{~s}$ ) or by filtering using different cut-off frequencies. Although this study attempted to control the start and end of the deceleration phase, it is possible that different approaches may lead to improved reliability and sensitivity. For example, using a 'start' and 'end' of deceleration phase criteria that is based on a deceleration threshold, such as, when deceleration is below and above $-0.2 \mathrm{~m} / \mathrm{s}^{2}$, respectively. Therefore, future research should investigate the reliability and sensitivity of different criteria that could be used to define the 'start' and 'end' of the deceleration phase. Furthermore, the radar device used in this study sampled at a rate of $47 \mathrm{~Hz}$. Other devices, such as lasers, capable of sampling at higher frequencies, may prove more reliable and sensitive to deceleration data. Additionally, low-cost, user friendly high speed video (capable of sampling at $240 \mathrm{~Hz}$ ), as used to profile sprint acceleration

472 performance and the associated mechanical outputs (Romero-Franco et al., 2017), could 473 be used to simultaneously gain important deceleration kinematic and kinetic data. The 474 simple computational methods used to calculate mechanical outputs have not been 475 validated and, subsequently, may therefore under- or over-estimate the actual values 476 reported. The participants used in this study were all young University sport athletes. 477 Research to investigate whether more experienced and higher performing (and perhaps 478 less variable) athletes demonstrate a greater level of assessment consistency is merited. 479 Also the horizontal acceleration-to-deceleration task used in this study was performed 
after one familiarisation session, and on an artificial indoor surface. Reliability and sensitivity of the data may, subsequently, be further improved when performed on sport-specific surfaces, or with more than one familiarisation session.

Finally, although the horizontal deceleration test used in the current study protocol requires multiple high intensity efforts, it replicates common team sport training tasks. Therefore, practitioners could implement this horizontal deceleration test into routine athlete monitoring systems, whilst also gaining performance and injury risk reduction benefits. Furthermore, simple adjustments to this deceleration test protocolfor example using different acceleration distances $(5,10$ and $15 \mathrm{~m})$ and prescribed distance targets, similar to those commonly used in COD tests (such as the 505), could provide an adaptive means to gather information on a diversity of deceleration tasks and abilities. Clearly, however, future research is needed to determine if the deceleration abilities assessed at lower horizontal velocities or momentums are reflective of the deceleration abilities assessed at higher horizontal velocities or momentums.

\section{Conclusions}

495 Using a novel maximal horizontal deceleration test, a number of radar derived

496 kinematic and kinetic variables had good intra-day reliability and were sufficiently sensitive to detect small-to-moderate worthwhile changes in deceleration performance. Only kinetic variables had good inter-day reliability, and were adequately able to detect moderate worthwhile changes in deceleration

500 performance after a single familiarisation session. Consequently, coaches and

501 sport science professionals can use mechanical outputs obtained from simple

502 computational methods to profile an individual's maximal horizontal deceleration

503 performance. In future, these approaches may provide insights illuminating the neuromuscular capabilities needed to decelerate maximally.

\section{Acknowledgements}

508 The authors would like to acknowledge the study participants and all the research assistants involved in the data collection process. 
512 Ashton, J., \& Jones, P. (2019). The reliability of using a laser device to assess deceleration ability. Sports, 7, 191. https://doi.org/10.3390/sports7080191

514 Cesar, G. M., \& Sigward, S. M. (2015). Dynamic stability during running gait termination: Differences in strategies between children and adults to control forward momentum. Human Movement Science, 43, 138-145. https://doi.org/10.1016/j.humov.2015.08.005

Cesar, G. M., \& Sigward, S. M. (2016). Dynamic stability during running gait termination: Predictors for successful control of forward momentum in children and adults. Human Movement Science, 48, 37-43. https://doi.org/10.1016/j.humov.2016.03.014

Colyer, S. L., Nagahara, R., Takai, Y., \& Salo, A. I. T. (2018). How sprinters accelerate beyond the velocity plateau of soccer players: Waveform analysis of ground reaction forces. Scandinavian Journal of Medicine and Science in Sports, 28(12), 2527-2535. https://doi.org/10.1111/sms.13302

Cronin, J. B., \& Templeton, R. L. (2008). Timing light height affects sprint times. Journal of Strength and Conditioning Research, 22(1), 318-320. https://doi.org/10.1519/JSC.0b013e31815fa3d3

Cross, M. R., Brughelli, M., Samozino, P., \& Morin, J.-B. (2017). Methods of powerforce-velocity profiling during sprint running: A narrative review. Sports Medicine, 47(7), 1255-1269. https://doi.org/10.1007/s40279-016-0653-3

Dalen, T., Ingebrigtsen, J., Ettema, G., Hjelde, G. H., \& Wisløff, U. (2016). Player load, acceleration, and deceleration during forty-five competitive matches of elite soccer. Journal of Strength and Conditioning Research, 30(2), 351-359. https://doi.org/10.1519/JSC.0000000000001063

de Hoyo, M., Cohen, D. D., Sañudo, B., Carrasco, L., Álvarez-Mesa, A., Del Ojo, J. J., ... Otero-Esquina, C. (2016). Influence of football match time-motion parameters on recovery time course of muscle damage and jump ability. Journal of Sports Sciences, 34(14), 1363-1370. https://doi.org/10.1080/02640414.2016.1150603

Dos'Santos, T., Thomas, C., Comfort, P., \& Jones, P. A. (2018). The effect of angle and velocity on change of direction biomechanics: An angle-velocity trade-off. Sports

543 Graham-Smith, P., Rumpf, M., \& Jones, P. (2018). Assessment of deceleration ability and relationship to approach speed and eccentric strength. ISBS-Conference 
Proceedings Archive, 36(1). https://doi.org/https://commons.nmu.edu/isbs/vol36/iss1/3/

Hader, K., Mendez-Villanueva, A., Palazzi, D., Ahmaidi, S., \& Buchheit, M. (2016). Metabolic power requirement of change of direction speed in young soccer players: Not all is what it seems. PLoS ONE, 11(3), e0149839. https://doi.org/10.1371/journal.pone.0149839

Hader, K., Palazzi, D., \& Buchheit, M. (2015). Change of direction speed in soccer: How much braking is enough? Kinesiology, 47(1), 67-74.

Harper, D. J., Carling, C., \& Kiely, J. (2019). High-intensity acceleration and deceleration demands in elite team sports competitive match play: A systematic review and meta-analysis of observational studies. Sports Medicine, 49(12), 19231947. https://doi.org/10.1007/s40279-019-01170-1

Harper, D. J., Jordan, A. R., \& Kiely, J. (2018). Relationships between eccentric and concentric knee strength capacities and maximal linear deceleration ability in male academy soccer players. Journal of Strength and Conditioning Research. https://doi.org/10.1519/JSC.0000000000002739

Harper, D. J., \& Kiely, J. (2018). Damaging nature of decelerations: Do we adequately prepare players? BMJ Open Sport \& Exercise Medicine, 4(1), e000379. https://doi.org/10.1136/bmjsem-2018-000379

Hopkins, W. G. (2015). Spreadsheets for analysis of validity and reliability. Sportscience, 19, 36-44. https://www.sportsci.org/2015/ValidRely.htm

Howatson, G., \& Milak, A. (2009). Exercise-induced muscle damage following a bout of sport specific repeated sprints. Journal of Strength and Conditioning Research, 23(8), 2419-2424. https://doi.org/10.1519/JSC.0b013e3181bac52e

Jian, Y., Winter, D., Ishac, M., \& Gilchrist, L. (1993). Trajectory of the body COG and COP during initiation and termination of gait. Gait \& Posture, 1(1), 9-22. https://doi.org/10.1016/0966-6362(93)90038-3 role of eccentric strength in $180^{\circ}$ turns in female soccer players. Sports, 5(2), 42. https://doi.org/10.3390/sports5020042 of Strength and Conditioning Research, 29(10), 2802-2807. https://doi.org/10.1519/JSC.0000000000000961 
Koo, T. K., \& Li, M. Y. (2016). A guideline of selecting and reporting intraclass correlation coefficients for reliability research. Journal of Chiropractic Medicine, 15(2), 155-163. https://doi.org/10.1016/j.jcm.2016.02.012

Loturco, I., Pereira, L. A., Freitas, T. T., Alcaraz, P. E., Zanetti, V., Bishop, C., \& Jeffreys, I. (2019). Maximum acceleration performance of professional soccer players in linear sprints: Is there a direct connection with change-of-direction ability? PLoS ONE, 14(5), e0216806. https://doi.org/10.1371/journal.pone.0216806

Martín-García, A., Casamichana, D., Gómez Díaz, A., Cos, F., \& Gabbett, T. J. (2018). Positional differences in the most demanding passages of play in football competition. Journal of Sports Science and Medicine, 17(4), 563-570.

McMahon, J., Lake, J., \& Comfort, P. (2018). Reliability of and relationship between flight time to contraction time ratio and reactive strength index modified. Sports, 6(3), 81. https://doi.org/10.3390/sports6030081

Morin, J.-B., Edouard, P., \& Samozino, P. (2011). Technical ability of force application as a determinant factor of sprint performance. Medicine and Science in Sports and Exercise, 43(9), 1680-1688. https://doi.org/10.1249/MSS.0b013e318216ea37

Morin, J.-B., Gimenez, P., Edouard, P., Arnal, P., Jiménez-Reyes, P., Samozino, P., ... Mendiguchia, J. (2015). Sprint acceleration mechanics: The major role of

Morin, J.-B., \& Samozino, P. (2016). Interpreting power-force-velocity profiles for individualized and specific training. International Journal of Sports Physiology and Performance, 11(2), 267-272. https://doi.org/10.1123/ijspp.2015-0638 method for computing sprint acceleration kinetics from running velocity data: Replication study with improved design. Journal of Biomechanics, 94, 82-87. https://doi.org/10.1016/j.jbiomech.2019.07.020

Nagahara, R., Botter, A., Rejc, E., Koido, M., Shimizu, T., Samozino, P., \& Morin, J.B. (2017). Concurrent validity of GPS for deriving mechanical properties of sprint acceleration. International Journal of Sports Physiology and Performance, 12(1), 129-132. https://doi.org/10.1123/ijspp.2015-0566 agility tests. The Journal of Sports Medicine and Physical Fitness, 55(11), 1329- 
Romero-Franco, N., Jiménez-Reyes, P., Castaño-Zambudio, A., Capelo-Ramírez, F., Rodríguez-Juan, J. J., González-Hernández, J., ... Balsalobre-Fernández, C. (2017). Sprint performance and mechanical outputs computed with an iPhone app: Comparison with existing reference methods. European Journal of Sport Science, 17(4), 386-392. https://doi.org/10.1080/17461391.2016.1249031

Russell, M., Sparkes, W., Northeast, J., Cook, C. J., Love, T. D., Bracken, R. M., \& professional soccer match-play. Journal of Strength and Conditioning Research, 30(10), 2839-2844. https://doi.org/10.1519/JSC.0000000000000805

Simperingham, K. D., Cronin, J. B., Pearson, S. N., \& Ross, A. (2019). Reliability of horizontal force-velocity-power profiling during short sprint-running accelerations using radar technology. Sports Biomechanics, 18(1), 88-99. https://doi.org/10.1080/14763141.2017.1386707

Simperingham, K. D., Cronin, J. B., \& Ross, A. (2016). Advances in sprint acceleration profiling for field-based team-sport athletes: Utility, reliability, validity and

Varley, M. C., Fairweather, I. H., \& Aughey, R. J. (2012). Validity and reliability of limitations. Sports Medicine, 46(11), 1619-1645. https://doi.org/10.1007/s40279-

Tierney, P. J., Young, A., Clarke, N. D., \& Duncan, M. J. (2016). Match play demands 016-0508-y 


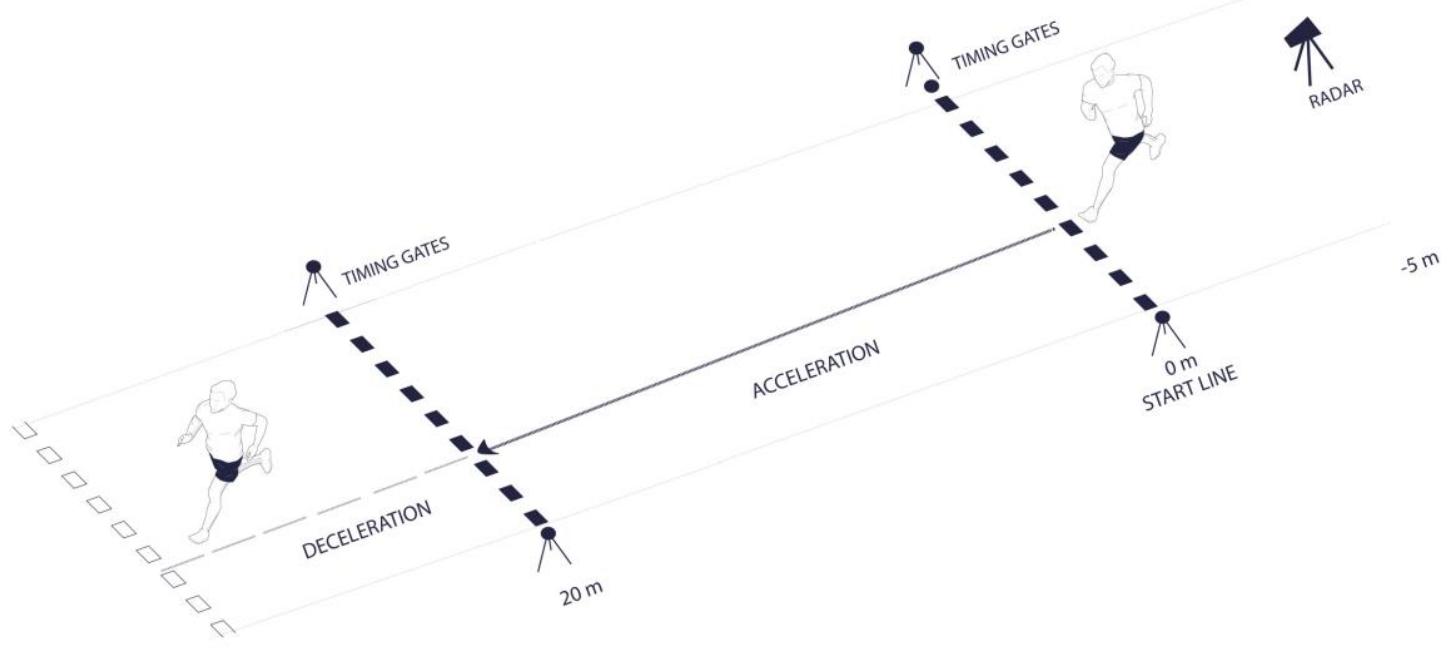

Figure 1. Acceleration-deceleration ability (ADA) test layout used to assess players maximal horizontal deceleration ability. 


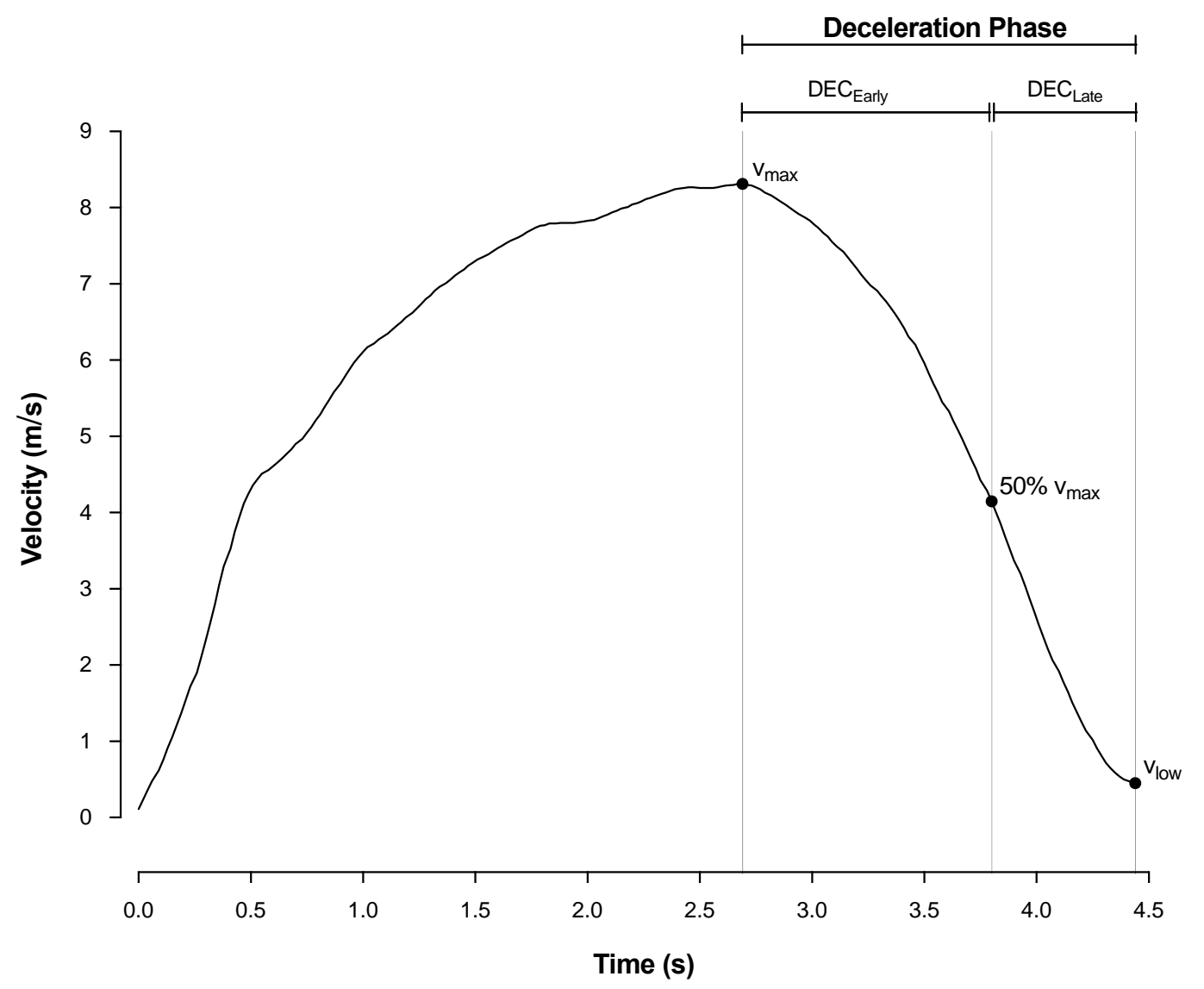

Figure 2. Example of velocity-time profile showing deceleration phase following manual processing with Stalker ATS ${ }^{\text {TM }}$ system software.

$V_{\max }=$ maximum velocity defining start of deceleration phase; $50 \% V_{\max }=50 \%$ of maximal velocity separating early and late deceleration phases; $V_{l o w}=$ lowest velocity defining end of deceleration phase; $\mathrm{DEC}_{\text {Early }}=$ early deceleration phase representing time between $V_{\max }$ and $50 \% V_{\max } ; \mathrm{DEC}_{\text {Late }}=$ late deceleration phase representing time between $50 \% V_{\max }$ and $V_{\text {low }}$ 
Table 1. Intra-day reliability and sensitivity of radar-derived kinematic and kinetic variables collected from the best 2 trials.

\begin{tabular}{|c|c|c|c|c|c|c|c|c|c|c|}
\hline \multirow[b]{2}{*}{ Variable } & \multirow[b]{2}{*}{ Trial 1} & \multirow[b]{2}{*}{ Trial 2} & \multicolumn{3}{|c|}{ Intra-day reliability } & \multicolumn{5}{|c|}{ Sensitivity } \\
\hline & & & ICC (90\% CL) & CV\% (90\% CL) & Rating & TE & $\mathrm{SWC}_{0.2}$ & Rating & $\mathrm{SWC}_{0.5}$ & Rating \\
\hline \multicolumn{11}{|l|}{ Kinematic } \\
\hline$V_{\max }(\mathrm{m} / \mathrm{s})$ & $7.34 \pm 0.55$ & $7.36 \pm 0.54$ & $0.97(0.95$ to 0.98$)$ & 1.4 (1.1 to 1.6$)$ & Excellent & 0.10 & 0.11 & Good & 0.27 & Good \\
\hline $\mathrm{TT} 50 \% V_{\max }(\mathrm{s})$ & $0.96 \pm 0.18$ & $0.98 \pm 0.13$ & $0.76(0.62$ to 0.85$)$ & $8.3(7.2$ to 10.3$)$ & Good & 0.08 & 0.03 & Marginal & 0.08 & $O K$ \\
\hline TTS (s) & $1.49 \pm 0.18$ & $1.51 \pm 0.17$ & $0.82(0.70$ to 0.89$)$ & $5.3(4.7$ to 6.7$)$ & Good & 0.08 & 0.04 & Marginal & 0.09 & Good \\
\hline DTS (m) & $6.78 \pm 1.06$ & $6.92 \pm 0.89$ & $0.76(0.61$ to 0.85$)$ & $7.2(6.0$ to 10.1$)$ & Good & 0.49 & 0.20 & Marginal & 0.49 & $O K$ \\
\hline $\operatorname{DEC}_{\text {ave }}\left(\mathrm{m} / \mathrm{s}^{2}\right)$ & $-4.45 \pm 0.61$ & $-4.44 \pm 0.62$ & $0.87(0.78$ to 0.92$)$ & $5.2(4.3$ to 6.3$)$ & Good & 0.23 & 0.12 & Marginal & 0.31 & Good \\
\hline E-DEC $C_{\text {ave }}\left(\mathrm{m} / \mathrm{s}^{2}\right)$ & $-3.89 \pm 0.72$ & $-3.86 \pm 0.63$ & $0.76(0.61$ to 0.85$)$ & $8.8(7.5$ to 10.8$)$ & Good & 0.34 & 0.13 & Marginal & 0.34 & $O K$ \\
\hline $\mathrm{L}-\mathrm{DEC}_{\text {ave }}\left(\mathrm{m} / \mathrm{s}^{2}\right)$ & $-5.57 \pm 0.79$ & $-5.62 \pm 0.78$ & $0.53(0.31$ to 0.70$)$ & 9.7 (8.2 to 12.0$)$ & Moderate & 0.54 & 0.16 & Marginal & 0.39 & Marginal \\
\hline $\mathrm{DEC}_{\max }\left(\mathrm{m} / \mathrm{s}^{2}\right)$ & $-8.50 \pm 1.07$ & $-8.46 \pm 1.30$ & $0.55(0.33$ to 0.71$)$ & $9.6(8.0$ to 11.8$)$ & Moderate & 0.81 & 0.24 & Marginal & 0.59 & Marginal \\
\hline $\mathrm{TTDEC}_{\max }(\mathrm{s})$ & $1.11 \pm 0.27$ & $1.15 \pm 0.22$ & $0.10(-0.17$ to 0.36$)$ & 20.4 (17.8 to 25.7$)$ & Poor & 0.23 & 0.05 & Marginal & 0.12 & Marginal \\
\hline \multicolumn{11}{|l|}{ Kinetic } \\
\hline $\mathrm{HBF}_{\text {ave }}(\mathrm{N})$ & $-318 \pm 81$ & $-318 \pm 78$ & $0.95(0.92$ to 0.97$)$ & $5.5(4.7$ to 6.9$)$ & Good & 17.6 & 15.9 & Marginal & 39.7 & Good \\
\hline $\mathrm{E}-\mathrm{HBF}_{\text {ave }}(\mathrm{N})$ & $-271 \pm 81$ & $-270 \pm 74$ & $0.89(0.82$ to 0.94$)$ & $9.6(8.1$ to 11.9$)$ & Good & 25.9 & 15.5 & Marginal & 38.7 & Good \\
\hline $\mathrm{L}-\mathrm{HBF}_{\text {ave }}(\mathrm{N})$ & $-406 \pm 90$ & $-407 \pm 98$ & $0.84(0.74$ to 0.91$)$ & 9.4 (7.9 to 11.7$)$ & Good & 38.3 & 18.9 & Marginal & 47.1 & Good \\
\hline $\mathrm{HBP}_{\text {ave }}(\mathrm{W})$ & $-1282 \pm 371$ & $-1273 \pm 370$ & $0.96(0.94$ to 0.98$)$ & 5.7 (4.8 to 7.0$)$ & Good & 72 & 74 & Good & 185 & Good \\
\hline E-HBP ave $(W)$ & $-1508 \pm 498$ & $-1500 \pm 479$ & $0.93(0.87$ to 0.96$)$ & $9.1(7.7$ to 11.3$)$ & Good & 137 & 98 & Marginal & 244 & Good \\
\hline L-HBP ave $(W)$ & $-927 \pm 229$ & $-907 \pm 248$ & $0.84(0.73$ to 0.90$)$ & $10.8(9.1$ to 13.3$)$ & Moderate & 99 & 48 & Marginal & 119 & Good \\
\hline $\mathrm{HBI}_{\mathrm{ave}}(\mathrm{N} / \mathrm{s})$ & $-6.81 \pm 1.71$ & $-6.80 \pm 1.65$ & $0.96(0.93$ to 0.98$)$ & $5.1(4.3$ to 6.3$)$ & Good & 0.35 & 0.34 & Marginal & 0.84 & Good \\
\hline E-HBI $\mathrm{Have}_{(\mathrm{N} / \mathrm{s})}$ & $-5.89 \pm 1.71$ & $-5.85 \pm 1.57$ & $0.91(0.84$ to 0.95$)$ & $8.7(7.3$ to 10.9$)$ & Good & 0.51 & 0.33 & Marginal & 0.82 & Good \\
\hline L-HBI $I_{\text {ave }}(\mathrm{N} / \mathrm{s})$ & $-8.52 \pm 1.89$ & $-8.55 \pm 2.05$ & $0.85(0.75$ to 0.91$)$ & $9.1(7.7$ to 11.4$)$ & Good & 0.78 & 0.39 & Marginal & 0.99 & Good \\
\hline $\mathrm{HBF}_{\max }(\mathrm{N})$ & $-616 \pm 137$ & $-610 \pm 149$ & $0.82(0.71$ to 0.89$)$ & 10.1 (8.6 to 12.6$)$ & Moderate & 62.1 & 28.6 & Marginal & 71.4 & Good \\
\hline $\mathrm{HBP}_{\max }(\mathrm{W})$ & $-2555 \pm 781$ & $-2544 \pm 713$ & $0.85(0.75$ to 0.91$)$ & 11.8 (9.9 to 14.6$)$ & Moderate & 301 & 150 & Marginal & 374 & Good \\
\hline $\mathrm{HBI}_{\max }(\mathrm{N} / \mathrm{s})$ & $-12.44 \pm 2.75$ & $-12.26 \pm 2.96$ & $0.83(0.72$ to 0.90$)$ & $9.8(8.3$ to 12.2$)$ & Good & 1.21 & 0.57 & Marginal & 1.43 & Good \\
\hline
\end{tabular}

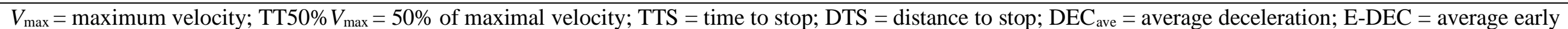

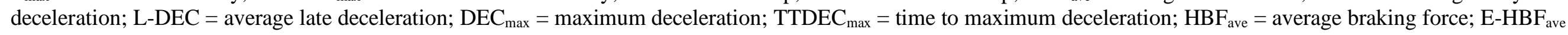

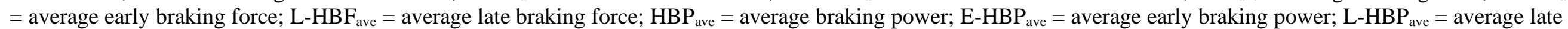

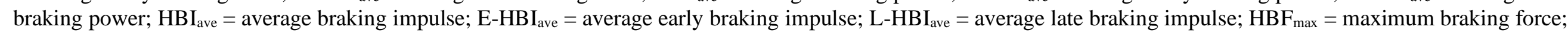
$\mathrm{HBP}_{\max }=$ maximum braking power; $\mathrm{HBI}_{\max }=$ maximum braking impulse . 
Table 2. Inter-day reliability and sensitivity of radar-derived kinematic and kinetic variables collected from the average of the best 2 trials, completed on 2 separate days of testing.

\begin{tabular}{|c|c|c|c|c|c|c|c|c|c|c|}
\hline \multirow[b]{2}{*}{ Variable } & \multirow[b]{2}{*}{ Day 1} & \multirow[b]{2}{*}{ Day 2} & \multicolumn{3}{|c|}{ Inter-test reliability } & \multicolumn{5}{|c|}{ Sensitivity } \\
\hline & & & ICC (90\% CL) & CV\% (90\% CL) & Rating & TE & $\mathrm{SWC}_{0.2}$ & Rating & $\mathrm{SWC}_{0.5}$ & Rating \\
\hline \multicolumn{11}{|l|}{ Kinematic } \\
\hline$V_{\max }(\mathrm{m} / \mathrm{s})$ & $7.19 \pm 0.54$ & $7.17 \pm 0.50$ & $0.96(0.88$ to 0.98$)$ & 1.7 (1.3 to 2.6$)$ & Excellent & 0.12 & 0.19 & Good & 0.46 & Good \\
\hline $\mathrm{TT} 50 \% V_{\max }(\mathrm{s})$ & $0.97 \pm 0.18$ & $0.96 \pm 0.13$ & $0.59(0.16$ to 0.83$)$ & 10.8 (8.1 to 16.8$)$ & Poor & 0.10 & 0.03 & Marginal & 0.08 & Marginal \\
\hline TTS (s) & $1.49 \pm 0.17$ & $1.47 \pm 0.14$ & 0.45 (-0.03 to 0.77$)$ & $8.2(6.1$ to 12.7$)$ & Moderate & 0.12 & 0.03 & Marginal & 0.08 & Marginal \\
\hline DTS (m) & $6.71 \pm 1.02$ & $6.53 \pm 0.83$ & $0.45(-0.03$ to 0.76$)$ & $10.8(8.0$ to 16.7$)$ & Poor & 0.71 & 0.19 & Marginal & 0.46 & Marginal \\
\hline $\mathrm{DEC}_{\mathrm{ave}}\left(\mathrm{m} / \mathrm{s}^{2}\right)$ & $-4.36 \pm 0.64$ & $-4.39 \pm 0.63$ & $0.73(0.40$ to 0.90$)$ & $8.0(6.0$ to 12.4$)$ & Moderate & 0.35 & 0.13 & Marginal & 0.32 & Marginal \\
\hline $\mathrm{E}-\mathrm{DEC}_{\mathrm{ave}}\left(\mathrm{m} / \mathrm{s}^{2}\right)$ & $-3.79 \pm 0.71$ & $-3.77 \pm 0.59$ & $0.55(0.10$ to 0.81$)$ & $12.1(9.0$ to 18.7$)$ & Poor & 0.46 & 0.13 & Marginal & 0.33 & Marginal \\
\hline $\mathrm{L}-\mathrm{DEC}_{\mathrm{ave}}\left(\mathrm{m} / \mathrm{s}^{2}\right)$ & $-5.55 \pm 0.60$ & $-5.53 \pm 0.70$ & $0.28(-0.23$ to 0.67$)$ & $10.1(7.6$ to 15.7$))$ & Poor & 0.56 & 0.13 & Marginal & 0.32 & Marginal \\
\hline $\mathrm{DEC}_{\max }\left(\mathrm{m} / \mathrm{s}^{2}\right)$ & $-8.27 \pm 0.91$ & $-8.40 \pm 1.07$ & 0.61 (0.19 to 0.84$)$ & $7.9(5.9$ to 12.2$)$ & Moderate & 0.65 & 0.20 & Marginal & 0.50 & Marginal \\
\hline $\mathrm{TTDEC}_{\max }(\mathrm{s})$ & $1.16 \pm 0.17$ & $1.17 \pm 0.17$ & $0.49(0.01$ to 0.78$)$ & $11.0(8.2$ to 17.1$)$ & Poor & 0.13 & 0.03 & Marginal & 0.09 & Marginal \\
\hline \multicolumn{11}{|l|}{ Kinetic } \\
\hline $\mathrm{HBF}_{\text {ave }}(\mathrm{N})$ & $-322 \pm 91$ & $-321 \pm 75$ & $0.90(0.73$ to 0.96$)$ & $9.3(7.0$ to 14.4$)$ & Good & 29.9 & 16.7 & Marginal & 41.8 & Good \\
\hline $\mathrm{E}-\mathrm{HBF}_{\text {ave }}(\mathrm{N})$ & $-273 \pm 91$ & $-273 \pm 70$ & $0.86(0.65$ to 0.95$)$ & $12.2(9.1$ to 18.9$)$ & Moderate & 33.2 & 16.2 & Marginal & 40.5 & Good \\
\hline $\mathrm{L}-\mathrm{HBF}_{\text {ave }}(\mathrm{N})$ & $-413 \pm 102$ & $-409 \pm 80$ & $0.76(0.45$ to 0.91$)$ & 11.7 (8.8 to 18.2$)$ & Moderate & 48.2 & 18.3 & Marginal & 45.8 & Marginal \\
\hline $\mathrm{HBP}_{\text {ave }}(\mathrm{W})$ & $-1272 \pm 414$ & $-1252 \pm 340$ & 0.93 (0.81 to 0.97$)$ & $8.9(6.6$ to 13.8$)$ & Good & 112 & 76 & Marginal & 189 & Good \\
\hline $\mathrm{E}-\mathrm{HBP}_{\mathrm{ave}}(\mathrm{W})$ & $-1490 \pm 550$ & $-1476 \pm 436$ & $0.89(0.73$ to 0.96$)$ & 12 (9.0 to 18.6$)$ & Moderate & 178 & 99 & Marginal & 248 & Good \\
\hline $\mathrm{L}-\mathrm{HBP}_{\mathrm{ave}}(\mathrm{W})$ & $-926 \pm 254$ & $-899 \pm 209$ & $0.66(0.26$ to 0.86$)$ & 21.6 (16.2 to & Poor & 259 & 83 & Marginal & 208 & Marginal \\
\hline $\mathrm{HBI}_{\mathrm{ave}}(\mathrm{N} / \mathrm{s})$ & $-6.87 \pm 1.93$ & $-6.86 \pm 1.59$ & $0.90(0.74$ to 0.96$)$ & $9.0(6.8$ to 14.0$)$ & Good & 0.62 & 0.35 & Marginal & 0.88 & Good \\
\hline $\mathrm{E}-\mathrm{HBI}_{\mathrm{ave}}(\mathrm{N} / \mathrm{s})$ & $-5.91 \pm 1.91$ & $-5.91 \pm 1.49$ & $0.87(0.67$ to 0.95$)$ & $11.6(8.6$ to 17.9$)$ & Moderate & 0.68 & 0.34 & Marginal & 0.86 & Good \\
\hline $\mathrm{L}-\mathrm{HBI}_{\mathrm{ave}}(\mathrm{N} / \mathrm{s})$ & $-8.68 \pm 2.13$ & $-8.59 \pm 1.70$ & $0.77(0.47$ to 0.91$)$ & $11.4(8.6$ to 17.7$)$ & Moderate & 0.99 & 0.39 & Marginal & 0.96 & Marginal \\
\hline $\mathrm{HBF}_{\max }(\mathrm{N})$ & $-616 \pm 149$ & $-623 \pm 134$ & $0.89(0.73$ to 0.96$)$ & $8.2(6.2$ to 12.8$)$ & Good & 51.0 & 28.4 & Marginal & 70.9 & Good \\
\hline $\mathrm{HBP}_{\max }(\mathrm{W})$ & $-2456 \pm 725$ & $-2372 \pm 627$ & $0.96(0.89$ to 0.99$)$ & $6.2(4.7$ to 9.7$)$ & Good & 151 & 136 & Marginal & 339 & Good \\
\hline $\mathrm{HBI}_{\max }(\mathrm{N} / \mathrm{s})$ & $-12.35 \pm 2.99$ & $-12.48 \pm 2.68$ & $0.90(0.73$ to 0.96$)$ & $8.2(6.1$ to 12.7$)$ & Good & 1.02 & 0.57 & Marginal & 1.42 & Good \\
\hline
\end{tabular}

$V_{\max }=$ maximum velocity; TT50\% $V_{\max }=50 \%$ of maximal velocity; TTS $=$ time to stop; DTS = distance to stop; DEC $\mathrm{Cve}_{\text {ave }}=$ average deceleration; E-DEC $=$ average early deceleration; $\mathrm{L}-\mathrm{DEC}=$ average late deceleration; $\mathrm{DEC}_{\max }=$ maximum deceleration; $\mathrm{TTDEC}_{\max }=$ time to maximum deceleration; $\mathrm{HBF}_{\mathrm{ave}}=$ average braking force; $\mathrm{E}$ $\mathrm{HBF}_{\text {ave }}=$ average early braking force; $\mathrm{L}-\mathrm{HBF}_{\text {ave }}=$ average late braking force; $\mathrm{HBP}_{\text {ave }}=$ average braking power; $\mathrm{E}-\mathrm{HBP}_{\text {ave }}=$ average early braking power; $\mathrm{L}-\mathrm{HBP}$ ave $=$ average late braking power; $\mathrm{HBI}_{\mathrm{ave}}=$ average braking impulse; $\mathrm{E}-\mathrm{HBI}_{\mathrm{ave}}=$ average early braking impulse; $\mathrm{L}-\mathrm{HBI}_{\mathrm{ave}}=$ average late braking impulse; $\mathrm{HBF}$ max $=$ maximum braking force; $\mathrm{HBP}_{\max }=$ maximum braking power; $\mathrm{HBI}_{\max }=$ maximum braking impulse. 
